\title{
Optimization of the firebox of a biomass-fired boiler
}

\author{
Tamás Tolnai * \\ Rail Safe Kft., 5051 Zagyvarékas, Csárda utca 31-43. Hungary.
}

Global Journal of Engineering and Technology Advances, 2021, 09(03), 105-113

Publication history: Received on 17 November 2021; revised on 22 December 2021; accepted on 24 December 2021

Article DOI: https://doi.org/10.30574/gjeta.2021.9.3.0170

\begin{abstract}
The most common fuel in crop drying is natural gas. Replacing this for renewable energy is both environmentally and economically beneficial. As a by-product of crop cleaning during harvesting, a source of energy suitable for combustion can be obtained from plant parts that are otherwise treated as waste. Solid fuel requires a special fuel system. Our goal is to find an optimized design for cleaning waste based on the existing solid fuel boilers, and in the framework of this work, the optimal design of the fire box passages is our narrower goal. To do this, we use CFD modelling, which is used to estimate the flow characteristics based on a 3D model in addition to the known operating parameters. By modifying the geometry between given boundaries, we find the ideal design within the boundaries allowed by the construction.
\end{abstract}

Keywords: Crop drying; By-product; Combustion; Solid fuel boiler; Firebox; CFD modelling

\section{Introduction}

As an alternative to fossil energy sources, biofuels have emerged in many areas. As a fuel for internal combustion engines for electricity generation or heating, for residential and industrial use [1, 2]. In richer countries, environmental protection, in poorer areas accessibility and price are the main reasons for using biomass. [1,3].

The main methods of converting solid biomass into energy are: direct combustion, pyrolysis, gasification $[3,4]$. Combustion is a common method of energy production because of its good adaptability and its ability to operate over a wider range. Appropriate pre-treatment (compaction, drying) can improve the efficiency of the method [5,6]. Fixed bed combustors are the most common solution as they are suitable for burning almost any solid fuel and are inexpensive to manufacture $[3,7,8]$. However, the technology also has drawbacks, one of which is the emission of particles and gases that are harmful to human health $[9,10]$. Fouling within the structure that degrade efficiency and can damage the internal structure due to their aggressive chemical properties [11,12].

By fluid dynamic modelling of the firebox, the internal physical state characteristics can be determined with sufficient accuracy, so that the combustion process can be planned. CFD (Computational Fluid Dynamics) methods are widely used for modelling and monitoring process behaviour $[13,14,15]$. These simulations are also suitable for testing and improving operation $[16,17]$.

\section{Fluid modelling of firebox geometry}

The aim of our work is to improve the combustion of agricultural by-products by modifying the geometry of the firebox. For the studies, we need a geometric model of the original combustion chamber. Using the combustion chamber model, we create a 3D model of the medium, which is the negative of the solid-state model (Fig. 1)

\footnotetext{
${ }^{*}$ Corresponding author: Tamás Tolnai

Rail Safe Kft., 5051 Zagyvarékas, Csárda utca 31-43. Hungary.

Copyright (C 2021 Author(s) retain the copyright of this article. This article is published under the terms of the Creative Commons Attribution Liscense 4.0.
} 


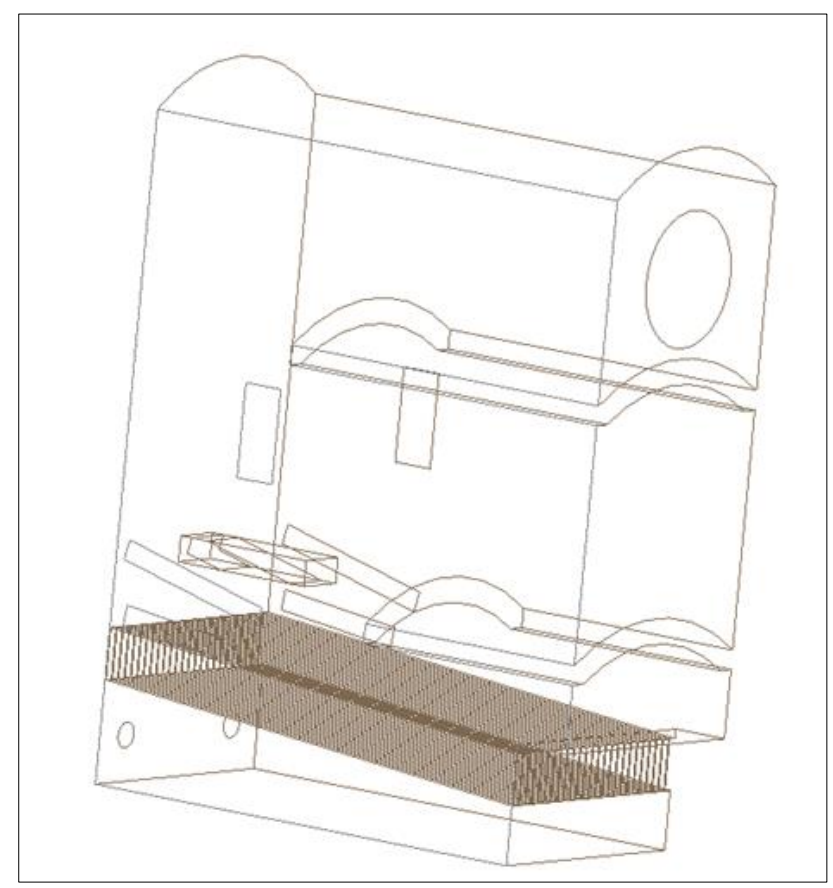

Figure $13 \mathrm{D}$ model of the fluid domain

During the modelling, a steady state is assumed. The flow and thermal characteristics of the medium are also taken into account, using an SST turbulence model.

Physical characteristics of the fluid domain:

- molar mass: $0,02896 \frac{\mathrm{kg}}{\mathrm{mol}}$

- $\quad$ specific heat: $4400 \frac{\mathrm{J}}{\mathrm{kg} \cdot \mathrm{K}}$

- dynamic viscosity: $1,831 * 10^{-5} \frac{\mathrm{kg}}{\mathrm{m} \cdot \mathrm{s}}$

- thermal conductivity: $0,0261 \frac{\mathrm{m}}{\mathrm{m} \cdot \mathrm{K}}$

- coefficient of thermal expansion: $0,003356 \frac{1}{K}$

The density of the medium was not treated as constant, considering that $\frac{V_{1}}{T_{1}}=\frac{V_{2}}{T_{2}}$, and $\rho_{1}=\frac{m}{V_{1}}$ also $\rho_{2}=\frac{m}{V_{2}}$ and using the value of the density of air at zero degrees, we finally obtain for the density function that

$\rho(T)=\frac{1}{354} T$

On the boundary surfaces of this model, the given environmental characteristics are defined as boundary conditions.

Boundary conditions for vaults: smooth, adiabatic walls, as they are not bounded by an external medium, only by an internal medium. The other boundary edges are in reality defined as solid, smooth walls, where the outside temperature is $20^{\circ} \mathrm{C}$, the heat transfer coefficient between the walls and the outside air $1 \mathrm{Wm}^{-2} \mathrm{~K}^{-1}$.

During the investigations, we looked for the effect of the geometrical characteristics of the model on the temperature and velocity conditions. To this end, the tests were performed with 30 different geometric modifications as defined in the following table (Table 1). 


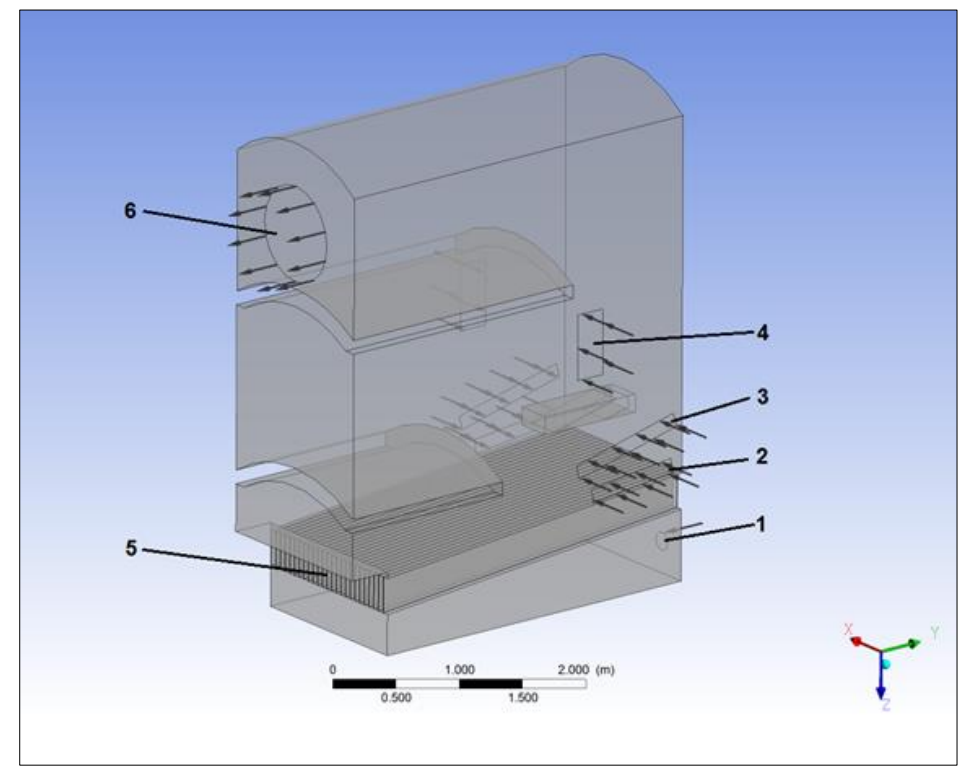

Figure 2 Boundary conditions

Boundary conditions for the lower blowers (1) are as follows. Subsonic gas mass flow $0.2257 \mathrm{kgs}^{-1}$ in a direction perpendicular to the surface, medium intensity, $5 \%$ turbulence. Inlet gas temperature $20^{\circ} \mathrm{C}$.

Boundary condition for the recirculation blowers (2): subsonic gas mass flow $0.11285 \mathrm{kgs}^{-1}$ in a direction perpendicular to the surface, medium intensity, $5 \%$ turbulence. Inlet gas temperature $1100{ }^{\circ} \mathrm{C}$.

Boundary condition for the second blowers (3): subsonic gas mass flow $0.2257 \mathrm{kgs}^{-1}$ in a direction perpendicular to the surface, medium intensity, $5 \%$ turbulence. Inlet gas temperature $1100{ }^{\circ} \mathrm{C}$.

Boundary condition for the third blowers (4): subsonic gas mass flow $0.11285 \mathrm{kgs}^{-1}$ in a direction perpendicular to the surface, medium intensity, $5 \%$ turbulence. Inlet gas temperature $1100{ }^{\circ} \mathrm{C}$.

Boundary condition for solid combustible material (5): smooth walls with a temperature of $1900{ }^{\circ} \mathrm{C}$.

Boundary condition for outlet (6): subsonic outflow, 0 Pa relative pressure.

Table 1 The investigated 30 geometric modifications of the firebox

\begin{tabular}{|c|c|c|c|c|c|c|c|c|c|c|c|}
\hline 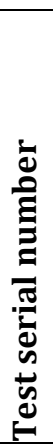 & 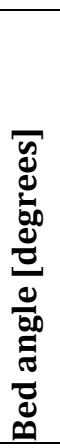 & 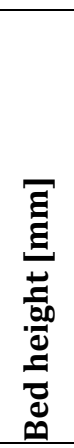 & 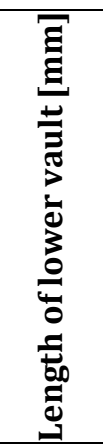 & 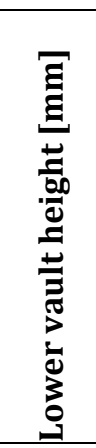 & 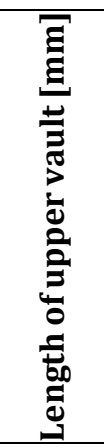 & 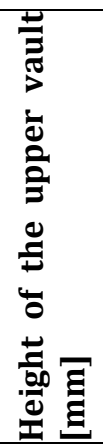 & 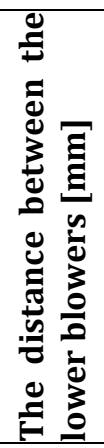 & 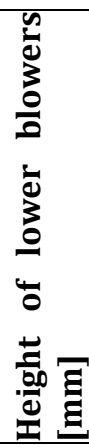 & 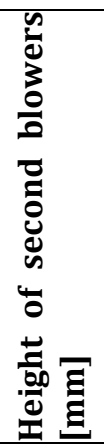 & 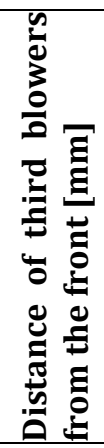 & 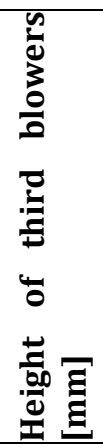 \\
\hline 1 & 5 & 600 & 1500 & 1100 & 2200 & 2600 & 1000 & 270 & 1170 & 800 & 1900 \\
\hline 2 & 3 & 600 & 1500 & 1100 & 2200 & 2600 & 1000 & 270 & 1170 & 800 & 1900 \\
\hline 3 & 1 & 600 & 1500 & 1100 & 2200 & 2600 & 1000 & 270 & 1170 & 800 & 1900 \\
\hline 4 & 5 & 400 & 1500 & 1100 & 2200 & 2600 & 1000 & 270 & 1170 & 800 & 1900 \\
\hline 5 & 5 & 500 & 1500 & 1100 & 2200 & 2600 & 1000 & 270 & 1170 & 800 & 1900 \\
\hline
\end{tabular}


Global Journal of Engineering and Technology Advances, 2021, 09(03), 105-113

\begin{tabular}{|c|c|c|c|c|c|c|c|c|c|c|c|}
\hline 6 & 5 & 600 & 1700 & 1100 & 2200 & 2600 & 1000 & 270 & 1170 & 800 & 1900 \\
\hline 7 & 5 & 600 & 1900 & 1100 & 2200 & 2600 & 1000 & 270 & 1170 & 800 & 1900 \\
\hline 8 & 5 & 600 & 1500 & 900 & 2200 & 2600 & 1000 & 270 & 1170 & 800 & 1900 \\
\hline 9 & 5 & 600 & 1500 & 1300 & 2200 & 2600 & 1000 & 270 & 1170 & 800 & 1900 \\
\hline 10 & 5 & 600 & 1500 & 1100 & 2000 & 2600 & 1000 & 270 & 1170 & 800 & 1900 \\
\hline 11 & 5 & 600 & 1500 & 1100 & 1800 & 2600 & 1000 & 270 & 1170 & 800 & 1900 \\
\hline 12 & 5 & 600 & 1500 & 1100 & 2200 & 2200 & 1000 & 270 & 1170 & 800 & 1900 \\
\hline 13 & 5 & 600 & 1500 & 1100 & 2200 & 2400 & 1000 & 270 & 1170 & 800 & 1900 \\
\hline 14 & 5 & 600 & 1500 & 1100 & 2200 & 2600 & 600 & 270 & 1170 & 800 & 1900 \\
\hline 15 & 5 & 600 & 1500 & 1100 & 2200 & 2600 & 800 & 270 & 1170 & 800 & 1900 \\
\hline 16 & 5 & 600 & 1500 & 1100 & 2200 & 2600 & 1000 & 170 & 1170 & 800 & 1900 \\
\hline 17 & 5 & 600 & 1500 & 1100 & 2200 & 2600 & 1000 & 370 & 1170 & 800 & 1900 \\
\hline 18 & 5 & 600 & 1500 & 1100 & 2200 & 2600 & 1000 & 270 & 1270 & 800 & 1900 \\
\hline 19 & 5 & 600 & 1500 & 1100 & 2200 & 2600 & 1000 & 270 & 1370 & 800 & 1900 \\
\hline 20 & 5 & 600 & 1500 & 1100 & 2200 & 2600 & 1000 & 270 & 1470 & 800 & 1900 \\
\hline 21 & 5 & 600 & 1500 & 1100 & 2200 & 2600 & 1000 & 270 & 1570 & 800 & 1900 \\
\hline 22 & 5 & 600 & 1500 & 1100 & 2200 & 2600 & 1000 & 270 & 1170 & 400 & 1900 \\
\hline 23 & 5 & 600 & 1500 & 1100 & 2200 & 2600 & 1000 & 270 & 1170 & 500 & 1900 \\
\hline 24 & 5 & 600 & 1500 & 1100 & 2200 & 2600 & 1000 & 270 & 1170 & 600 & 1900 \\
\hline 25 & 5 & 600 & 1500 & 1100 & 2200 & 2600 & 1000 & 270 & 1170 & 700 & 1900 \\
\hline 26 & 5 & 600 & 1500 & 1100 & 2200 & 2600 & 1000 & 270 & 1170 & 800 & 1500 \\
\hline 27 & 5 & 600 & 1500 & 1100 & 2200 & 2600 & 1000 & 270 & 1170 & 800 & 1600 \\
\hline 28 & 5 & 600 & 1500 & 1100 & 2200 & 2600 & 1000 & 270 & 1170 & 800 & 1700 \\
\hline 29 & 5 & 600 & 1500 & 1100 & 2200 & 2600 & 1000 & 270 & 1170 & 800 & 1800 \\
\hline 30 & 5 & 600 & 1500 & 1100 & 2200 & 2600 & 1000 & 270 & 1170 & 800 & 2000 \\
\hline
\end{tabular}

\section{Results}

Material and heat flow conditions inside the firebox were determined using numerical flow modelling methods.

\subsection{Velocity conditions}

First we examine the flow and temperature conditions of the basic structure presented above for the dynamic equilibrium state.

Figure 3 shows the flow pattern of the flue gases in the boiler. An important goal is to find a design where flue gases also enter between the two vaults, allowing for even heating.

In the following, we can see the velocity distributions for each plane section in several consecutive figures.

Figure 4 shows the flow velocity conditions above the plane of the second inlets. Here, the velocity values are, of course, lower than in the immediate vicinity of the recirculation inlet, but the flow field in the firebox is visibly more uniform. 


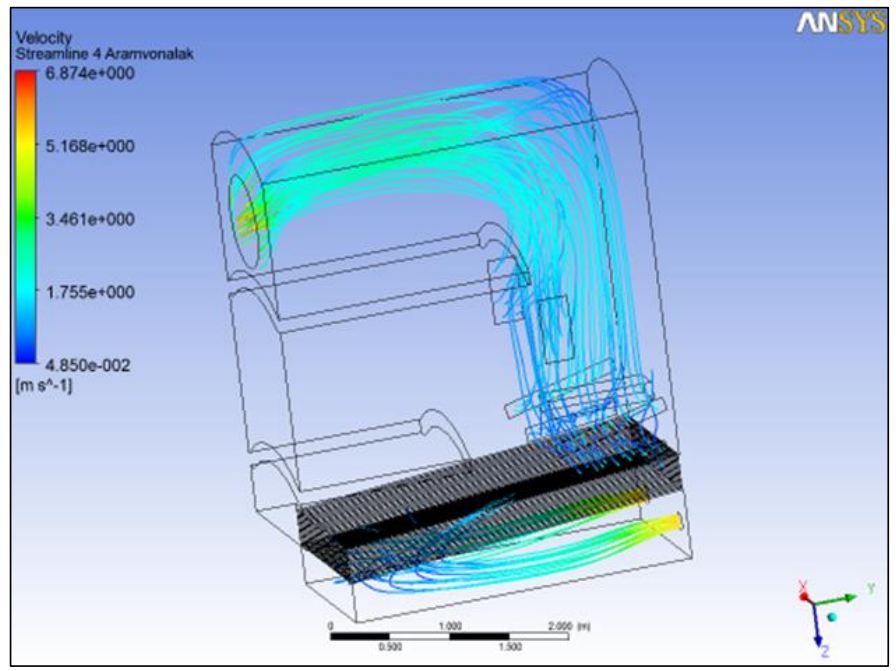

Figure 3 Streamlines in the firebox

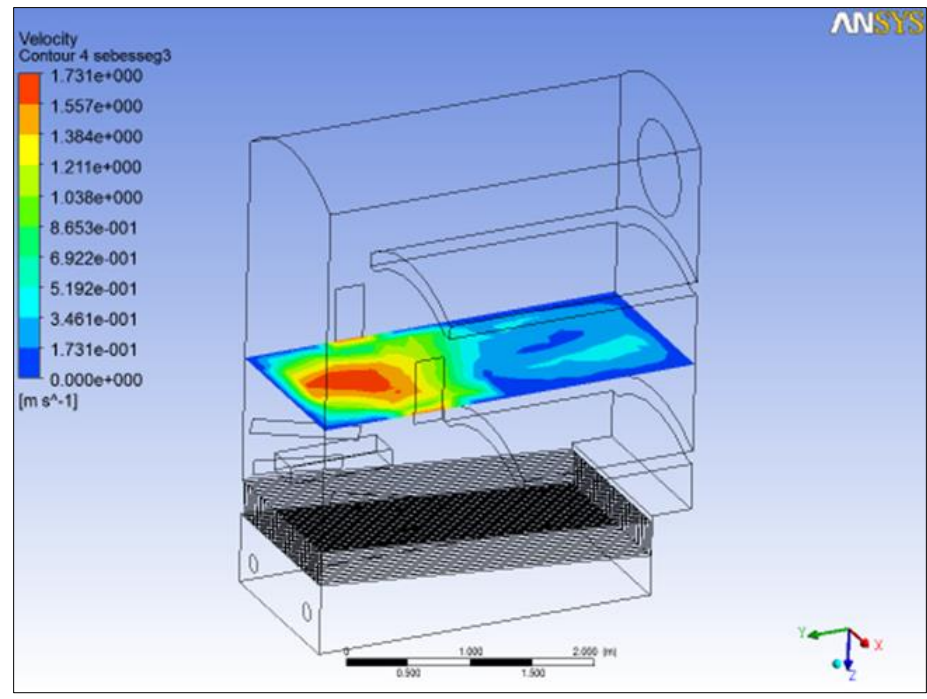

Figure 4 Flow velocity conditions in the plane of the third inlet

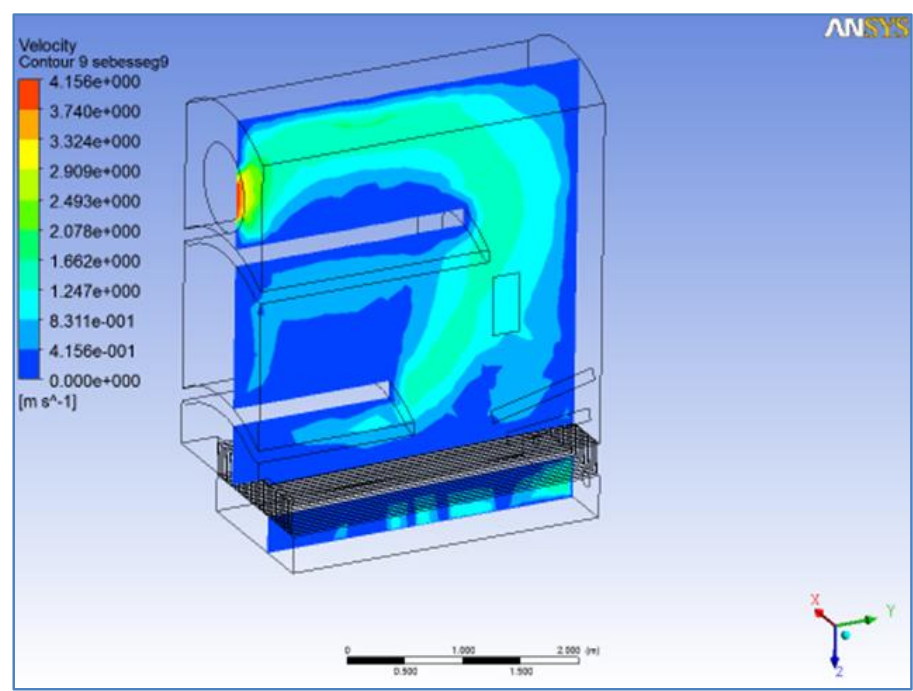

Figure 5 Velocity conditions in the selected vertical section of the firebox 
Here, too, it is important that hot gases flow wherever possible around the vault, allowing for efficient heat transfer

Figure 5 shows the velocity conditions in a vertical section $100 \mathrm{~mm}$ from the plane of symmetry of the firebox. As this section shows the part of the boiler as far away as possible from the inlets, we find low flow rate values in the vicinity of the grate. Of course, the velocity conditions at the outlet well reflect the high velocity values that develop there.

\subsection{Temperature conditions}

The temperature conditions of the basic structure are examined in two planar sections. In the horizontal plane of the upper air vents and in the horizontal plane above the upper vault.

As the boiler moves upwards (away from the direct heat source), the maximum temperature decreases, the overall temperature distribution inside the boiler becomes more uniform and the minimum temperature increases.

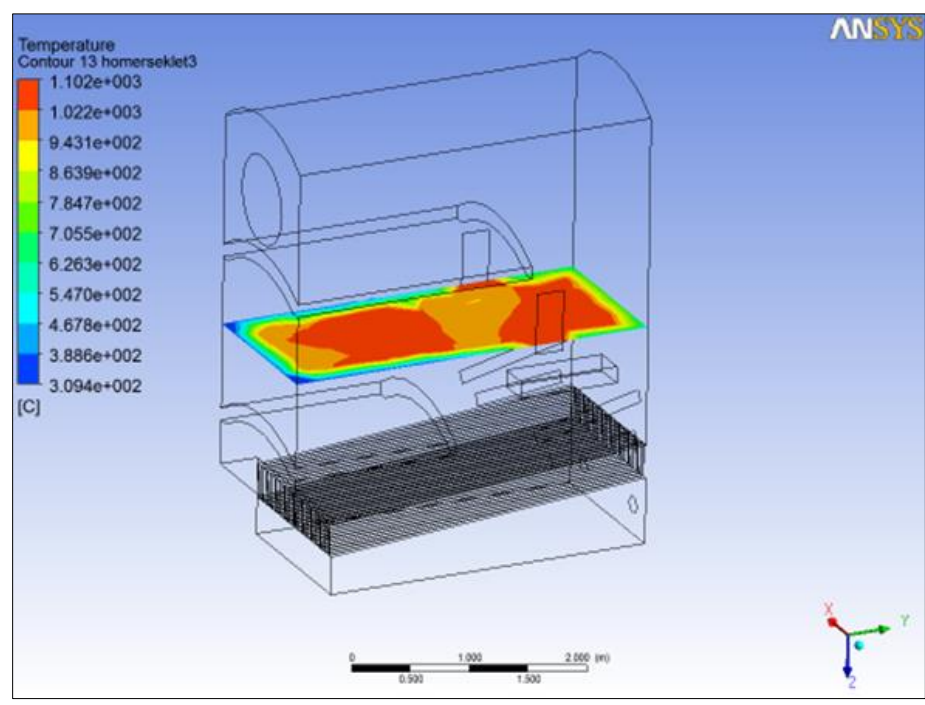

Figure 6 Temperature distribution at the height of the upper blowers

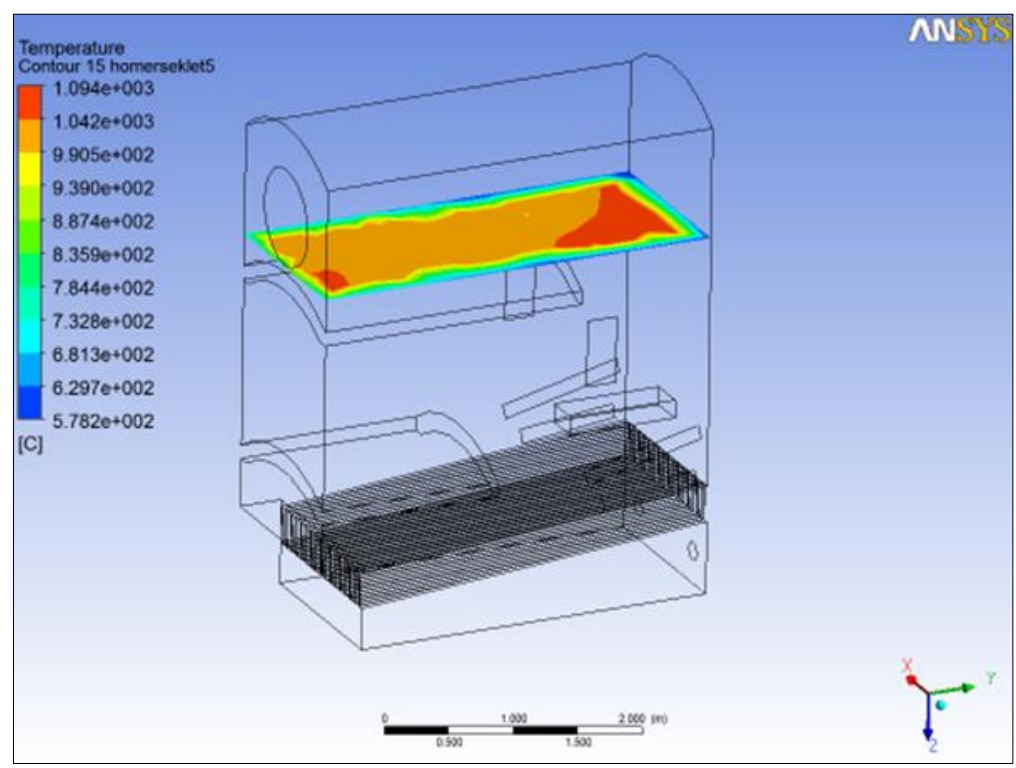

Figure 7 Temperature distribution above the second vault

The effect of different geometric modifications on the selected test values is shown in the following table (Table 2). 
Table 2 Maximum speed and temperature according to the 30 different modifications

\begin{tabular}{|c|c|c|c|c|}
\hline Set & $\begin{array}{l}\text { Maximum speeds } \\
\text { according to Figure } 4\end{array}$ & $\begin{array}{l}\text { Maximum speeds } \\
\text { according to Figure } 5\end{array}$ & $\begin{array}{l}\text { Maximum temperatures } \\
\text { according to Figure } 6\end{array}$ & $\begin{array}{l}\text { Maximum temperatures } \\
\text { according to Figure } 7\end{array}$ \\
\hline 1 & 1.203 & 4.7 & 964.7 & 742.9 \\
\hline 2 & 1.334 & 4.156 & 925.6 & 790.7 \\
\hline 3 & 1.184 & 4.069 & 926.8 & 767.7 \\
\hline 4 & 1.565 & 4.374 & 914.6 & 806.3 \\
\hline 5 & 1.254 & 4.17 & 914.1 & 762.4 \\
\hline 6 & 1.327 & 4.025 & 927.2 & 740.4 \\
\hline 7 & 1.366 & 4.071 & 939.4 & 740.5 \\
\hline 8 & 1.22 & 3.966 & 913.1 & 735.6 \\
\hline 9 & 1.119 & 4.044 & 924.4 & 745.9 \\
\hline 10 & 1.249 & 4.09 & 924.3 & 738.5 \\
\hline 11 & 1.143 & 4.063 & 930.7 & 765.7 \\
\hline 12 & 1.216 & 3.873 & 875.1 & 715.7 \\
\hline 13 & 1.223 & 3.937 & 895.2 & 717.4 \\
\hline 14 & 1.269 & 6.083 & 889.6 & 712.2 \\
\hline 15 & 1.171 & 4.331 & 906.1 & 769 \\
\hline 16 & 1.386 & 4.053 & 930.3 & 733.2 \\
\hline 17 & 1.419 & 4.226 & 892.9 & 751.8 \\
\hline 18 & 1.221 & 4.213 & 993.4 & 785.5 \\
\hline 19 & 1.238 & 4.12 & 989.4 & 753.2 \\
\hline 20 & 1.204 & 3.913 & 922.4 & 738.5 \\
\hline 21 & 1.181 & 4.22 & 920.5 & 752.8 \\
\hline 22 & 1.226 & 4.112 & 915.7 & 759.8 \\
\hline 23 & 1.175 & 4.077 & 985.8 & 792.1 \\
\hline 24 & 1.186 & 4.039 & 918.7 & 767.9 \\
\hline 25 & 1.218 & 4.037 & 919.1 & 761.3 \\
\hline 26 & 1.315 & 4.299 & 931.3 & 757.5 \\
\hline 27 & 1.353 & 4.07 & 928 & 749.5 \\
\hline 28 & 1.193 & 3.998 & 924.3 & 744.1 \\
\hline 29 & 1.327 & 4.128 & 920.6 & 745.9 \\
\hline 30 & 1.193 & 4.051 & 928.7 & 741.6 \\
\hline
\end{tabular}

\section{Conclusion}

Examining the results, it can be seen that the velocity in the lower and upper planes changed only slightly, with one exception, at speed 14, the velocity is remarkably high, which is caused by the approximation of the lower blowers and should be avoided. The outlet temperature values are shown in Figure 8 for ease of reference. 


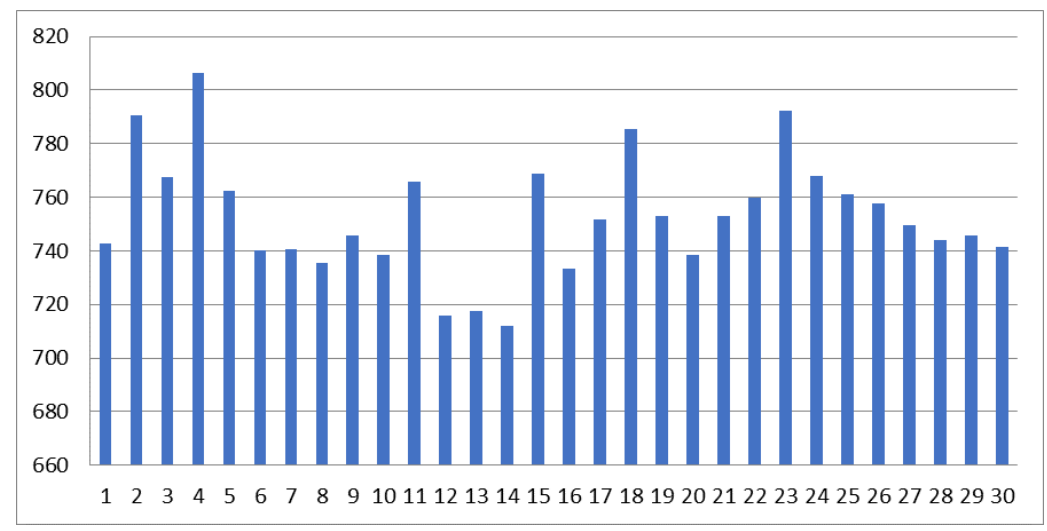

Figure 8 Temperature maximum values in the horizontal plane above the upper vault

Based on Figure 8, we find two extreme positions. The maximum is for setting 4, the minimum is for settings 12,13 (14 is excluded due to too high a speed). Based on this, it can be concluded that the lower position of the grate causes the highest upper temperatures. The minimum temperatures occur when the height of the upper vault is reduced.

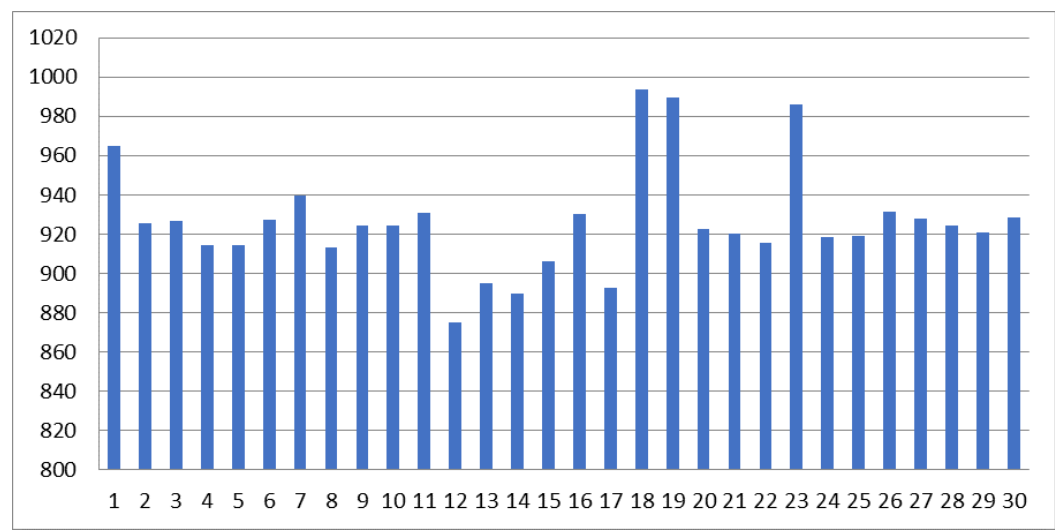

Figure 9 Temperature maximum values in the horizontal plane of the upper blower

In the middle of the firebox, we find three extremely high temperature values, with settings $18,19,23$. Because the combustion process is important in this region, high temperatures are preferred. This can be achieved by changing the position of the second and third blowers by positioning them differently from the original.

\section{Compliance with ethical standards}

\section{Acknowledgments}

The author appreciates the assistance of his colleagues during the research project.

\section{Disclosure of conflict of interest}

The author has declared that there is no existence of competing interests.

\section{References}

[1] World Energy Council, Bioenergy, World Energy Resources. 2016.

[2] Derek W. Bunn, Jorge Redondo-Martin, José I. Muñoz-Hernandez, Pablo Diaz- Cachinero, Analysis of coal conversion to biomass as a transitional technology, Renew. Energy. 2019; 132: 752-760.

[3] Richard L Bain, Ralph P. Overend, Kevin R. Craig, Biomass-fired power generation, Fuel Process. Technol. 1998; 54: 1-16. 
[4] Jorge Islas, Fabio Manzini, Omar Masera, Viridiana Vargas, Chapter four - solid biomass to heat and power, in: Carmen Lago, Natalia Caldés, Yolanda Lechón (Eds.), The Role of Bioenergy in the Emerging Bioeconomy, Resources, Technologies, Sustainability and Policy. 2019; 145-177.

[5] Priyabrata Pradhan, Sanjay M Mahanaji, Amit Arora. Production and utilization of fuel pellets from biomass: a review, Fuel Process. Technol. 2018; 181: 215-232.

[6] D Patiño, J Moran, J Porteiro, J Collazo, E Granada, JL Miguez. Improving the cofiring process of wood pellet and refuse derived fuel in a small-scale boiler plant, Energy Fuel. 2018; 22: 2121-2128.

[7] Suzan Abdelhady, Domenico Borello, Ahmed Shaban, Techno-economic assessment of biomass power plant fed with rice straw: sensitivity and parametric analysis of the performance and the LCOE, Renew. Energy. 2018; 115: 1026-1034.

[8] Esa Kari Vakkilainen, 9 - direct and grate firing of biomass, in: Esa Kari Vakkilainen, Steam Generation from Biomass, Construction and Design of Large Boilers. 2017; 203-210.

[9] EJS Mitchell, AR Lea-Langton, JM Jones, A Williams, P Layden, R Johnson. The impact of fuel properties on the emissions from the combustion of biomass and other solid fuels in a fixed bed domestic stove, Fuel Process. Technol. 2016; 142: 115-123.

[10] Zhongfa Hu, Xuebin Wang, Adewale Adeosun, Renhui Ruan, Houzhang Tan, Aggravated fine particulate matter emissions from heating-upgraded biomass and biochar combustion: the effect of pretreatment temperature, Fuel Process. Technol. 2018; 171: 1-9.

[11] Araceli Regueiro, David Patiño, Enrique Granada, Jacobo Porteiro, Experimental study on the fouling behaviour of an underfeed fixed-bed biomass combustor, Appl. Therm. Eng. 2017; 112: 523-533.

[12] Joaquín Capablo, Formation of alkali salt deposits in biomass combustion, Fuel Process. Technol. 2016; 153: 5873.

[13] MA Gómez, J Porteiro, S Chapela, JL Míguez. An Eulerian model for the simulation of the thermal conversion of a single large biomass particle, Fuel. 2018; 220: 671-681.

[14] Anqi Zhou, Hongpeng Xu, Yaojie Tu, Feiyang Zhao, Zhiming Zheng, Wenming Yang, Numerical investigation of the effect of air supply and oxygen enrichment on the biomass combustion in the grate boiler, Appl. Therm. Eng. 2019; 156: 550-561.

[15] CA Bermúdez, Jacobo Porteiro, Luis G Varela, Sergio Chapela, David Patiño, Three-dimensional CFD simulation of a large-scale grate-fired biomass furnace, Fuel Processing Technology. 2020; 198: 106219.

[16] Adeline Rezeau, Luis I Díez, Javier Royo, Maryori Díaz-Ramírez, Efficient diagnosis of grate-fired biomass boilers by a simplified CFD-based approach, Fuel Process. Technol. 2018; 171: 318-329.

[17] Anqi Zhou, Hongpeng Xu, Yaojie Tu, Feiyang Zhao, Zhiming Zheng, Wenming Yang, Numerical investigation of the effect of air supply and oxygen enrichment on the biomass combustion in the grate boiler, Appl. Therm. 